\title{
POTENTIOMETRIC TITRATION AND CONFORMATION OF SYNTHETIC AND NATURAL POLYELECTROLYTES
}

\author{
Misturu NAgasawa \\ Department of Synthetic Chemistry, Nagoya University, \\ Chikusa-ku, Nagoya, Japan
}

\begin{abstract}
From the analysis of the potentiometric titration data of polyelectrolytes, we can obtain the electrostatic free energy of the macromolecules. Therefore, potentiometric titration is a useful experimental procedure to study the conformation and conformational change of macromolecules. A few examples of the application of potentiometric titration to the studies on polypeptides, protein and DNA are discussed. When the potentiometric titration method is not applicable, the numerical solution of the Poisson-Boltzmann equation without use of the Debye-Hückel approximation may be employed to estimate the electrostatic potential at the surface of macromolecules. To confirm the reliability in the values thus estimated without experiments and in the values of the electrostatic free energy calculated from the surface potential, the calculated potentiometric titration data are compared with experimental data of various polyelectrolytes and proteins. The relationship between potentiometric titration data and conformations of stereoregular polyelectrolytes is also discussed.
\end{abstract}

\section{INTRODUCTION}

A carboxylic acid in aqueous solution dissociates as

$$
-\mathrm{COOH} \rightleftharpoons-\mathrm{COO}^{-}+\mathrm{H}^{+}
$$

Its thermodynamic dissociation constant, $K_{a}$, is expressed by

$$
K_{a}=\left(-\mathrm{COO}^{-}\right)\left(\mathrm{H}^{+}\right) /(-\mathrm{COOH})
$$

where parentheses denote the activity of each species. Often, however, the conventional dissociation constant, $K_{0}$, defined by

$$
K_{0}=\left[-\mathrm{COO}^{-}\right]\left(\mathrm{H}^{+}\right) /[-\mathrm{COOH}]
$$

is used instead of $K_{a}$ since we do not know the activities (in parentheses) of $-\mathrm{COO}^{-}$and $-\mathrm{COOH}$ but only their analytical concentrations (in brackets). Therefore, $K_{0}$ is not always a true constant, while $K_{a}$ must be.

Equation $2^{\prime}$ can be written

$$
\mathrm{pH}=\mathrm{p} K_{0}-\log [(1-\alpha) / \alpha]
$$




\section{MISTURU NAGASAWA}

where the degree of ionization $\alpha$ is defined by

$$
\alpha=\left[-\mathrm{COO}^{-}\right] /\left\{\left[-\mathrm{COO}^{-}\right]+[-\mathrm{COOH}]\right\}
$$

Ignoring the distinction between equations 2 and $2^{\prime}, \mathrm{p} K_{0}$ may be related to the standard free energy change of the dissociation process, $\Delta G^{0}$, as

$$
\mathrm{p} K_{0}=-\log K_{0}=0.434 \Delta G^{0} / R T
$$

In the dissociation of polyelectrolytes ${ }^{1-7}$, however, an additional amount of work, $\Delta G_{\mathrm{el}}$, is required; i.e. it is necessary to remove $\mathrm{H}^{+}$against the strong electrostatic forces of the charges already present in the molecule. That is, the apparent ionization constant, $\mathrm{p} K$, of a polyacid, which is defined by equation 6, comprises two terms; the intrinsic standard free energy change $\Delta G^{0}$ and the additional electrostatic free energy change $\Delta G_{\mathrm{el}}$.

$$
\begin{aligned}
\mathrm{p} K & \equiv \mathrm{pH}+\log [(1-\alpha) / \alpha] \\
& =0.434\left(\Delta G^{0}+\Delta G_{\mathrm{el}}\right) / R T
\end{aligned}
$$

Thus, equation 3 must be modified to read

$$
\mathrm{pH}=\mathrm{p} K_{0}-\log [(1-\alpha) / \alpha]+0.434 \Delta G_{\mathrm{el}} / R T
$$

Since $\Delta G_{\mathrm{el}}$ is the additional electric work to remove a hydrogen ion from the surface of the macro-ion to infinity, $\Delta G_{\mathrm{el}}$ is equal to the change of the electrostatic free energy, $G_{\mathrm{e} 1}$, of the macro-ion when the charge of the macromolecule is increased by one

$$
\Delta G_{\mathrm{e} 1}=\partial G_{\mathrm{el}}(Z) / \partial Z
$$

where $-Z$ is the protonic charge number of the macro-ion. Thus, we have

$$
G_{\mathrm{el}}(\alpha) / N=2.3 R T \int_{0}^{\alpha}\left\{\mathrm{pH}+\log [(1-\alpha) / \alpha]-\mathrm{p} K_{0}\right\} \mathrm{d} \alpha
$$

where $\alpha=Z / N, N$ being the total number of ionizable groups in the molecule, or the degree of ionization. Equation 10 means that the electrostatic free energy of a polyacid having the degree of ionization $\alpha, G_{\mathrm{el}}(\alpha) / N$, is given by the area under the graph of $\mathrm{pH}+[\log (1-\alpha) / \alpha]-\mathrm{p} K_{0}$ versus $\alpha$.

Alternatively, $\Delta G_{\mathrm{el}}$ can be expressed by

$$
\Delta G_{\mathrm{el}}=N_{\mathrm{A}} e \psi_{\mathrm{b}}
$$

in terms of the electrostatic potential at the place where $\mathrm{H}^{+}$originally existed, $\psi_{\mathrm{b}}$. Therefore, $G_{\mathrm{el}}(\alpha) / N$ can also be calculated from

$$
G_{\mathrm{e} 1}(\alpha) / N=R T \int_{0}^{\alpha} \phi_{\alpha} \mathrm{d} \alpha
$$

where

$$
\phi_{\mathrm{b}}=e \psi_{\mathrm{b}} / k T
$$

Equation 12 means that the electrostatic free energy of a macro-ion can be calculated by charging up the molecule from the uncharged state to the actual state of ionization degree $\alpha$. Equations 10 and 12 are expected to give 


\section{SYNTHETIC AND NATURAL POLYELECTROLYTES}

the same result if we can calculate $\phi_{\mathrm{b}}$ as a function of $\alpha$. That is, titration is an experimental procedure of charging up a molecule.

The reduced potential $\phi$ around a polyacid which is in an infinite volume of a simple electrolyte solution of uni-uni valence type can be obtained by solving the following Poisson-Boltzmann equation.

$$
\begin{gathered}
\nabla^{2} \psi=-(4 \pi / D) \rho \\
\rho=\left(N_{A} e C_{S} / 10^{3}\right)[\exp (-e \psi / k T)-\exp (e \psi / k T)]
\end{gathered}
$$

The solution of equation 14 is usually obtained on the Debye-Hückel approximation, i.e. $e \psi\left(k T \ll 1\right.$; by Linderstrom-Lang ${ }^{8,9}$ for spherical proteins and by Hill ${ }^{10}$ for rodlike molecules. However, it has often been pointed out that the Debye-Hückel approximation is inapplicable not only to highly charged linear polyelectrolytes but also to charged proteins, ionic polypeptides or DNA. In this work, therefore, the solution of equation 14 obtained by numerical integration without using the Debye-Hückel approximation $^{11,12}$ is used for discussion.

Since we can obtain the electrostatic free energy of a macro-ion from potentiometric titration data, the potentiometric titration has a particular importance in studying the conformational change of macromolecules. A well known example of the application of potentiometric titration in this field may be the study of the helix-coil transition of ionic polypeptides.

\section{APPLICATION OF POTENTIOMETRIC TITRATION TO THE STUDY OF CONFORMATIONAL CHANGE OF MACROMOLECULES}

\section{(1) Ionic polypeptides}

As has been demonstrated by many investigators, a typical potentiometric titration curve of ionic polypeptides such as poly(L-glutamic acid) is as shown in Figure $1^{13}$. Let us assume that there is a molecule of poly(L-glutamic acid)

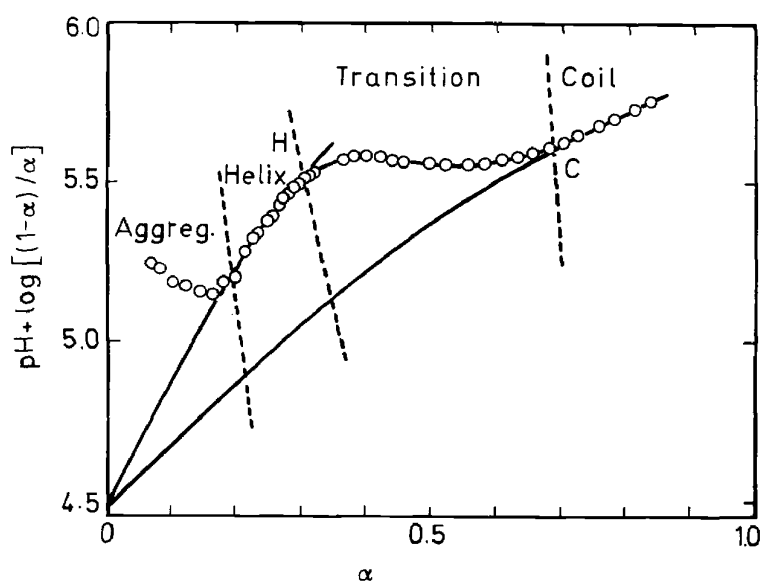

Figure 1. An example of the potentiometric titration curve of poly(L-glutamic acid). $0.0342 \mathrm{~N}$ PGA, $0.02 \mathrm{M} \mathrm{NaCl}$. (Reproduced, by permission, from ref. 12) 
which has zero degree of ionization and a helical conformation. The molecule is represented by the symbol $O$ in Figure 1. If the molecule is titrated with a caustic alkali, the potentiometric titration data lie along the line $\mathrm{OH}$ and the electrostatic free energy of the molecule increases in proportion to the area under the curve $0 \mathrm{H}$. After a certain amount of electrostatic free energy is accumulated on the helical molecule, further increase of the electrostatic free energy is consumed to change the conformation of $\alpha$-helix to random coil and the electrostatic free energy of the molecule increases along the curve HC. After the molecule becomes a complete coil, the coil is discharged prohibiting re-formation of the helix. Then, the electrostatic free energy decreases along the line $\mathrm{C}$, and finally the molecule becomes an uncharged coil. The total electric work given to the molecule in this cycle is given by the area $0 \mathrm{HC}$ and the resultant reaction is the transition of an uncharged helix to an uncharged coil. Therefore, the area $0 \mathrm{HC}$ gives the standard free energy change in the helix-coil transition of charged poly(L-glutamic acid), $\Delta G_{\alpha}^{\infty} / N, N$ being the number of monomers in the molecule. More detailed discussions on this problem are given by Zimm and Rice ${ }^{14}$, by Leyte and Mandel $^{14 a}$ and by Nagasawa and Holtzer ${ }^{13}$.

Examples of the non-electrostatic part of the free energy change for the helix-coil transitions of ionic polypeptides are shown in Table $1^{15-19,13}$.

Table 1. Non-electric part of the standard free energy change for the helix-coil transition of polypeptides

\begin{tabular}{lcl}
\hline \multicolumn{1}{c}{ Polypeptide } & $\Delta G_{a}^{\infty} / N(\mathrm{cal} / \mathrm{mol})$ & Reference \\
\hline glutamic acid & $100 \sim 300$ & $13,15,16$ \\
lysine & $100 \sim 150$ & 17 \\
leucine & 840 & 18 \\
alanine & $0 \sim 50$ & 19 \\
\hline
\end{tabular}

Except the value for poly(leucine $)^{18}$, the value per hydrogen bond is at most a few hundreds of calories. The values for non-ionic polypeptides were determined by preparing copolymers with an ionic peptide.

\section{(2) DNA ${ }^{20}$}

In neutral solutions, DNA has a double-stranded helix and each phosphate group on the molecule has a negative charge. If the solution is heated, DNA is denatured into two coiled molecules, on which, too, each phosphate group has a negative charge. That is, the melting occurs as

$$
h^{-z} \rightleftharpoons 2 c^{-Z / 2}
$$

The total free energy change for the melting of DNA is made up of two terms. One is the electrostatic free energy change, $\Delta G_{\mathrm{el}}^{\infty} / N_{\mathrm{p}}\left(N_{\mathrm{p}}\right.$ being the number of base pairs in the molecule), which is given as the difference between the electrostatic free energy of the original helix and that of two coiled molecules. The other is the remaining part of the free energy change, that is, the nonelectrostatic free energy change $\Delta G_{a}^{\infty} / N_{\mathrm{p}}$. The non-electrostatic free energy 


\section{SYNTHETIC AND NATURAL POLYELECTROLYTES}

change may include the energy of the hydrogen bond, the stacking of base pairs and the entropy of coils, etc. That is, the total free energy change $\Delta G^{\infty} / N_{\mathrm{p}}$ is

$$
\Delta G^{\infty} / N_{\mathrm{p}}=\Delta G_{a}^{\infty} / N_{\mathrm{p}}+\Delta G_{\mathrm{el}}^{\infty} / N_{\mathrm{p}}
$$

The non-electrostatic free energy change is favourable for the structure of a double-stranded helix, whereas the electrostatic free energy change tends to destroy this structure.

The electrostatic free energy of the double-stranded helix is calculated as the work to charge up the rod from the uncharged state to the actual state, as explained in the introduction. The calculation of the electrostatic free energy of a coiled polynucleotide, i.e. that of coiled polyelectrolytes, has not fully been clarified because of the change of its conformation during the charging up process. In this paper, we assume that the electrostatic free energy of a coiled polyelectrolyte may be calculated approximately as the work to charge up the polyion skeleton, assuming the polyion to be a rodlike molecule. That is, it is assumed that the electrostatic free energy of a coiled polyelectrolyte is accumulated around the skeleton ${ }^{21}$. This assumption will be discussed later.

The $\Delta G_{a}^{\infty} / N_{\mathrm{p}}$ in equation 16 is believed to be more or less independent of ionic strength, but $\Delta G_{\mathrm{el}}^{\infty} / N_{\mathrm{p}}$ varies with ionic strength. Consequently, the total free energy change in the melting, $\Delta G^{\infty} / N_{\mathrm{p}}$, also depends on ionic strength. Recently, the total enthalpy change and, consequently, the total free energy change per base pair at $37^{\circ} \mathrm{C}, \Delta G^{\circ} / N_{\mathrm{p}}$, was determined in solutions of various ionic strengths by Privalov, Ptitsyn and Birnstein ${ }^{22}$ using a microcalorimeter. Their values, $\Delta G^{\infty} / N_{\mathrm{p}}$, and also $\Delta G_{\mathrm{el}}^{\infty} / N_{\mathrm{p}}$ calculated by the above method at various ionic strengths are listed in Table 2 . The values

Table 2. Non-electric part of the free energy change for the melting of DNA. Sample, guaninezytosine content $=33$ per cent, at $37^{\circ} \mathrm{C} . \Delta G^{\infty}$, by Privalov et al., Biopolymers, 8, 559 (1969).

\begin{tabular}{ccccc}
\hline $\begin{array}{c}C_{\mathrm{s}} \\
(\mathrm{mol} / 1 .)\end{array}$ & $\begin{array}{c}\Delta G^{\infty} / N \mathrm{p} \\
\text { (cal) }\end{array}$ & $\begin{array}{c}\Delta G_{\mathrm{el}}^{\infty} / N_{\mathrm{p}} \\
\text { (cal) }\end{array}$ & $\begin{array}{c}\Delta G_{a}^{\infty} / N_{\mathrm{p}} \\
\text { (cal) }\end{array}$ & $\begin{array}{c}\Delta G_{a}^{c} / N_{\mathrm{h}} \\
\text { (cal) }\end{array}$ \\
\hline 0.205 & 1250 & -924 & 2170 & 930 \\
0.115 & 1180 & -1084 & 2260 & 970 \\
0.057 & 1020 & -1287 & 2310 & 990 \\
0.036 & 910 & -1417 & 2310 & 1000 \\
0.014 & 780 & -1682 & 2460 & 1060 \\
0.011 & 710 & -1756 & 2470 & 1060 \\
\hline
\end{tabular}

Av. 1000

of $\Delta G_{a}^{\infty} / N_{\mathrm{p}}$ can then be calculated from those values as also shown in Table 2.

It may be concluded from Table 2 that the ionic strength dependence of $\Delta G^{\infty} / N_{\mathrm{p}}$ is almost independent of ionic strength as speculated. If we calculate the non-electrostatic free energy change per hydrogen bond $\Delta G_{a}^{\infty} / N_{\mathrm{h}}$ from $\Delta G_{a}^{\infty} / N_{\mathrm{p}}$ by taking into account the guanine-cytosine content of the DNA, $\Delta G_{a}^{\infty} / N_{\mathrm{h}}$ is found to be about $1.0 \mathrm{kcal}$, which is considerably higher than the non-electrostatic free energy change per hydrogen bond for the helix-coil 
transition of synthetic polypeptides. The high value of $\Delta G_{a}^{\infty} / N_{\mathrm{h}}$ for DNA appears to imply that the stacking energy of base pairs is important for the stability of DNA.

It is well known that the denaturation of DNA does not occur as an allor-nothing process, but occurs as an intramolecular helix-random coil transition. At the transition point, each molecule has both helical and coiled portions. Therefore, it is assumed in this work that the electrostatic free energy of the double-stranded helix per base pair in a DNA molecule is equal to that of a perfect double-stranded helix and that of a coiled portion is equal to that of coiled polynucleotides. There are various theories on the mechanism of melting of $\mathrm{DNA}^{23-26}$. In most theories, the degree of helix in DNA $\theta$ is given in terms of a parameter $s$, the statistical weight of a base pair in the double-stranded helix relative to that in the denatured state, i.e. the equilibrium constant for the process of increasing a bonded sequence by one base pair. Although different theories give somewhat different dependence of $\theta$ upon $s, \theta$ decreases to zero sharply with decrease of $s$ at a critical value of $s$ which is close to unity.

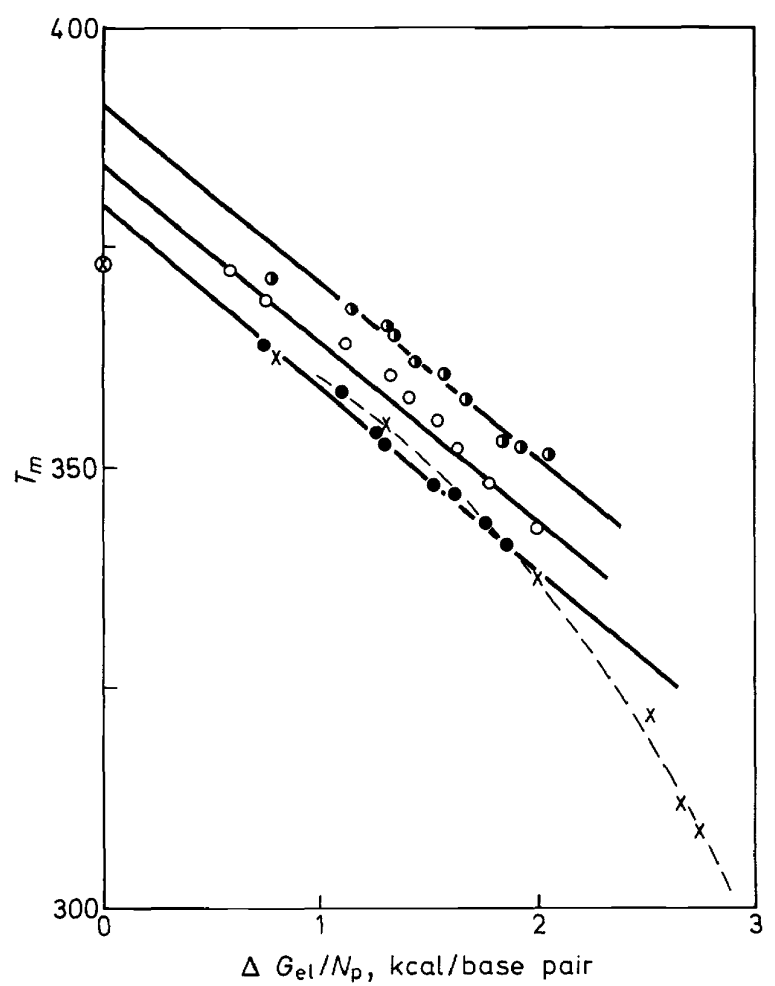

Figure 2. The linear relationship ${ }^{20}$ between the melting temperature $T_{m}$ and the electrostatic free energy of melting $\Delta G_{\mathrm{el}}^{\infty} / N_{\mathrm{p}}$. Filled circles, $D$. pneumoniae $(\mathrm{G}-\mathrm{C} \text { content } 39 \%)^{28}$. Open circles, E. coli $(\mathrm{G}-\mathrm{C} \text { content } 50 \%)^{28}$. Half-filled circles, Ps. aeruginosa. $(\mathrm{G}-\mathrm{C} \text { content } 60 \%)^{29}$. Cross, B. megaterium (G-C content $37 \%)^{30}$. Cross in a circle, Calf thymus $(\mathbf{G}-\mathrm{C}$ content $44 \%$ ) plus poly(lysine $)^{31}$ 


\section{SYNTHETIC AND NATURAL POLYELECTROLYTES}

If there is no electrostatic effect, $s$ is given by ${ }^{26}$

$$
s_{0}=g \exp \left(\Delta H / N_{\mathrm{p}} R T\right)
$$

where $\Delta H / N_{\mathrm{p}}$ is the heat of dissociation of a base pair and $g$ is a constant which depends on the structure of a nucleotide. If there is an electrostatic effect in the dissociation of a base pair, $s$ should be modified as ${ }^{27}$

$$
s=s_{0} \exp \left(\Delta G_{\mathrm{el}}^{\infty} / N_{\mathrm{p}} R T\right)
$$

At the melting temperature, we may assume $s \simeq 1$ and have

$$
T_{m}=T_{m}^{0}+A \Delta G_{\mathrm{el}}^{\infty} / N_{\mathrm{p}}
$$

where $T_{m}^{0}$ is the melting temperature of uncharged DNA and a constant $A$ is

$$
A=[-R \ln g]^{-1}
$$

In Figure 2, $T_{m}$ reported by various authors ${ }^{28-30}$ is plotted against $\Delta G_{\mathrm{el}}^{\infty} / N_{\mathrm{p}}$ calculated by the above method. As expected, $T_{m}$ is found to be linear with respect to $\Delta G_{\mathrm{el}}^{\infty} / N_{\mathrm{p}}$ if the ionic strength is not very high. The melting temperature of uncharged DNA, $T_{m}^{0}$, can be obtained as the limit of $\Delta G_{\mathrm{el}}^{\infty} / N_{\mathrm{p}}=0$. It is found to be $106^{\circ}, 110^{\circ}$ and $118^{\circ} \mathrm{C}$ for DNA of 39,50 and 60 per cent guanine-cytosine contents, respectively. These values are in fair agreement with $T_{m}^{0}=c a \cdot 100^{\circ} \mathrm{C}$ for a neutral DNA of 44 per cent guanine-cytosine content, which is prepared by combining polylysine on the molecule ${ }^{31}$.

\section{(3) B-Lactoglobulin ${ }^{32}$}

Let us assume that a protein molecule is in equilibrium with two identical subunits at any given $\mathrm{pH}$ and ionic strength, and also that both the dimers and the subunit are spherical and have charges $+Z$ and $+Z / 2$ protonic units, respectively. That is,

$$
P_{2}^{+Z} \rightleftharpoons 2 P^{+Z / 2}
$$

The protein $\beta$-lactoglobulin displays such a dissociation equilibrium, and it has been the subject of extensive experimental investigation by Timasheff, Townend and their co-workers ${ }^{33,34}$. By determining the weight average molecular weight, it is possible to assess the weight fraction of protein present as dimer and as monomer and thus to determine the numerical value of the 'macroscopic' equilibrium constant for dissociation

$$
K=4 \beta^{2} C /(1-\beta)
$$

where $C$ is the total formality of protein (formula weight 35500 , i.e. as dimer) and $\beta$ is the weight fraction of subunits (monomer) in the solution. The standard free energy change for this process is calculated using the measured $K$ and the relation

$$
\Delta G^{\infty}=-R T \ln K
$$

As in the melting of DNA, $\Delta G^{\infty}$ may be divided into two parts, an electrostatic part $\left(\Delta G_{\mathrm{el}}^{\infty}\right)$ and a remainder $\left(\Delta G_{a}^{\infty}\right)$ :

$$
\Delta G^{\infty}=\Delta G_{a}^{\infty}+\Delta G_{\mathrm{el}}^{\infty}
$$


The values of $\Delta G^{\infty}$ for $\beta$-lactoglobulin at different $\mathrm{pH}$ and ionic strengths are given by Timasheff $e t a l .{ }^{33}$ as listed in Table 3. The values of $\Delta G_{\mathrm{el}}^{\alpha}$ listed in Table 3 are calculated by using the charging up method, assuming that the dissociation is isochoric, i.e. $a=26.2 \AA$ for dimer and $26.2 / \sqrt[3]{ } 2 \AA$ for a subunit. The numbers of charges on the molecule at different $\mathrm{pH}$ and ionic strengths were determined by a potentiometric titration method. From $\Delta G^{\infty}$

Table 3. $\Delta G_{a}^{\infty}(\mathrm{kcal} / \mathrm{r}$.u.) of $\beta$-lactoglobulin

\begin{tabular}{|c|c|c|c|c|c|c|}
\hline \multirow[t]{2}{*}{$\mathrm{NaCl}$ conc. } & \multicolumn{6}{|c|}{$0.1 \mathrm{M}$} \\
\hline & 1.6 & 2.0 & 2.5 & 2.7 & 3.0 & 3.5 \\
\hline$Z$ & 39.1 & 38.4 & 36.3 & 34.8 & 31.6 & 24.4 \\
\hline $\begin{array}{l}\Delta G^{\infty} \\
\text { by Timasheff } e t \text { al. }\end{array}$ & 4.9 & 4.9 & 5.5 & 5.7 & 5.8 & 7.3 \\
\hline$-\Delta G_{\mathrm{el}}^{\infty}$ & 5.7 & 5.6 & 5.1 & 4.2 & 3.9 & 2.6 \\
\hline$\Delta G_{a}^{\circ}$ & 10.6 & 10.5 & 10.6 & 10.3 & 9.7 & 9.9 \\
\hline $\mathrm{NaCl}$ conc. & $0.03 \mathrm{M}$ & $0.3 \mathrm{M}$ & & & & \\
\hline $\mathrm{pH}$ & 2.7 & 2.7 & & & & \\
\hline$Z$ & 33.0 & 36.8 & & & & \\
\hline $\begin{array}{l}\Delta G^{\infty} \\
\text { by Timasheff } e t \text { al. }\end{array}$ & 3.9 & 8.3 & & & & \\
\hline$-\Delta G_{\mathbf{e l}}^{\infty}$ & 6.9 & 4.0 & & & & \\
\hline$\Delta G_{a}^{\infty}$ & 10.8 & 12.3 & Av. 10 & \pm 1 & & \\
\hline
\end{tabular}

and $\Delta G_{\mathrm{el}}^{\infty}$, we can have $\Delta G_{a}^{\infty}$ which are almost independent of $\mathrm{pH}$ and ionic strength, as seen in Table 3, the average value being $+10.6 \pm 1 \mathrm{kcal} / \mathrm{r}$.u. Unfortunately, we have little to say about this value, since the chemical groupings on the surface of contact between the subunits are not known.

\section{COMPARISON BETWEEN THE COMPUTED ELECTROSTATIC POTENTIAL AND THE POTENTIOMETRIC TITRATION DATA}

In the above discussions, we used the caicuiated electrostatic potential to estimate the electrostatic free energy. A method to confirm the reliability of the calculated electrostatic potential at the surface may be to compare the calculated values with the experimental data of potentiometric titration. That is, $\mathrm{pH}$ in solutions of a polyelectrolyte is related to the electrostatic potential at the surface by

$$
\mathrm{pH}+\log [(1-\alpha) / \alpha]-\mathrm{p} K_{0}=0.434 \phi_{\mathrm{b}}
$$

The validity of $\phi_{\mathrm{b}}$ used to calculate the electrostatic free energy of the molecule can therefore be tested by comparing the computed $\phi_{\mathrm{b}}$ with experimental values of $\mathrm{pH}+\log [(1-\alpha) / \alpha]-\mathrm{p} K_{0}$. For this comparison, however, two 
ambiguous points must be clarified. One is that the electrostatic potential calculated from the Poisson-Boltzmann equation is the potential at the radius $(a)$ to which the simple ions are excluded, $\phi_{a}$, whereas the potential required for calculation, of the electrostatic free energy is the potential at the surface $(b)$ of the macro-ion, $\phi_{b}$. There is the following relationship ${ }^{12}$ between $\phi_{a}$ and $\phi_{b}$

$$
\phi_{b}=\phi_{a}+B Z
$$

where $\mathbf{B}=\left(e^{2} / D k T\right)[(a-b) / a b]$ for a spherical macro-ion

$$
=\left(2 e^{2} / D k T\right) \ln (a / b) \text { for a rodlike macro-ion }
$$

The calculated value of $\phi_{b}$ is not sensitive to the choice of $a$ or $b$, but is sensitive to the choice of $a-b$. The difference $a-b$ should represent the radius of the salt ion, e.g. about $2.5 \AA$ for a sodium ion. Strictly speaking, however, the location of the protons may not coincide exactly with the radius of the molecule. The only practical way is to treat $B$ as an adjustable parameter and choose the value of $B$ that fits the data best. Additionally, we do expect the value of $a-b$ needed to produce agreement to be roughly of the dimension of a salt ion (i.e. 1 to $2 \AA$ ). When calculating the electrostatic free energy change, the difference between $\phi_{a}$ and $\phi_{b}$ was neglected because this term is almost cancelled between the original molecule and denatured molecules.

The other problem is the ionic strength dependence of $\mathrm{p} K_{0}$. From equations 2 and $2^{\prime}$, we know $\mathrm{p} K_{0}$ should not be constant but should depend on ionic strength, though $\mathrm{p} K_{a}$ is a constant independent of ionic strength. In this work, it is assumed that the ionic strength dependence of $\mathrm{p} K_{0}$ is parallel to the ionic strength dependence of the activity coefficient of the monobasic carboxyl group in the salt solution. That is, assuming that the activity coefficients of potassium and chloride ions in $\mathrm{KCl}$ solutions are equal and also that the activity coefficient of an ion is constant if the ionic strength is constant, the ionic strength dependence of $-\mathrm{COO}^{-}$may be speculated from those of the activity coefficients of various monovalent carboxylic acids $^{35}$. The ionic strength dependence of $\mathrm{pK}_{0}$ was experimentally discussed by Olander and Holtzer ${ }^{15}$ and by Mandel ${ }^{36}$.

\section{(1) Spherical macro-ions ${ }^{32}$}

The experimental values of $\mathrm{pH}+\log [(1-\alpha) / \alpha]$ for $\beta$-lactoglobulin in sodium chloride solutions are plotted against $Z$ in Figure 3 and are compared with those calculated from theoretical values of $\phi_{b}$. In $0.5 \mathrm{M} \mathrm{NaCl}$ solution, there is no dissociation of $\beta$-lactoglobulin and, therefore, the agreement between the observed values of $\mathrm{pH}+\log [(1-\alpha) / \alpha]$ and the calculated values for undissociated $\beta$-lactoglobulin is very good though the constant $B$ must be chosen arbitrarily. In $0.01 \mathrm{M} \mathrm{NaCl}$, however, there is a considerable amount of dissociation of $\beta$-lactoglobulin at high charge densities.

Using $\Delta G_{\mathrm{el}}^{\infty}$ calculated by the charging up method together with $\Delta G_{a}^{\infty}=$ $10.6 \mathrm{kcal} / \mathrm{r}$.u., we obtain $\Delta G^{\infty}$ and then $\beta$ at any charge density. Therefore, the value of $\phi_{b}$ for an equilibrium mixture of monomer and dimer is determined as the weighted numerical average of the values for monomer and dimer. The values of $\phi_{b}$ thus calculated are shown by solid lines in Figure 3 


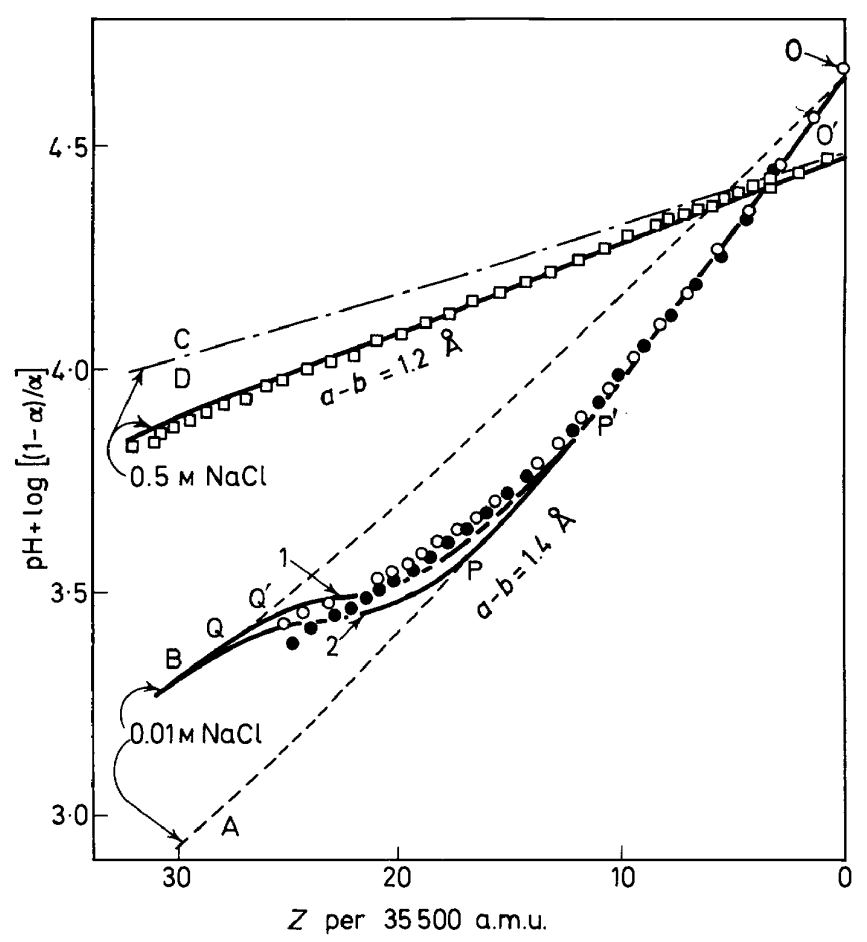

Figure 3. Potentiometric titration curves for the carboxyl groups of $\beta$-lactoglobulin. Theoretical curves $\mathrm{O}^{\prime} \mathrm{C}$ and $\mathrm{O}^{\prime} \mathrm{D}$ are for dimer in $0.5 \mathrm{M} \mathrm{NaCl}$ with $a=26.2 \AA$. For curve $\mathrm{O}^{\prime} \mathrm{C}, a-b=0$; for $\mathrm{O}^{\prime} \mathrm{D}, a-b=1.2 \AA$. Open squares are data points for $0.469 \%$ protein in $0.5 \mathrm{M} \mathrm{NaCl}$. Dashed curve OP'PA (OQQ ${ }^{\prime} B$ ) is calculated from theory for dimer (subunit) in $0.01 \mathrm{M} \mathrm{NaCl}$ with $a=$ $26.2 \AA(26.2 / \sqrt[3]{2} \AA)$ and $a-b=1.4 \AA$. Solid curves are those calculated as described in the text for mixtures of dimer and subunits using the dashed curves as a basis. The upper solid curve (1) is for $0.194 \%$ protein, the lower (2) for $0.469 \%$. Open circles denote experimental points for $0.194 \%$ protein and filled circles for $0.469 \%$ in $0.01 \mathrm{M} \mathrm{NaCl}$. (Reproduced, by permission, from ref. 12)

for comparison with experimental values of $\mathrm{pH}+\log [(1-\alpha) / \alpha]$. If we thus take dissociation into account, we find that $a-b=1.4 \AA$ provides the best fit with experimental results. Although agreement is still not perfect, the potentiometric titration data of a spherical protein are well expressed by the calculated curves, at least up to $Z=25$. The remaining disagreement between the theoretical curves and experimental points may arise mainly from our simple assumption on the distribution of charges between dimer and subunits as shown in equation $21^{32}$.

Based on the same principle as employed for the helix-coil transition of poly(L-glutamic acid), $\Delta G_{a}^{\infty}$ can be determined from the area defined by the experimental points and the subunit curve, though the graph for the subunit is simply a computed one. The only difference between these cases is that, here, the reaction is a bimolecular one, that is, an uncharged dimer is converted to two molecules of uncharged subunits after one cycle of electrical work, whereas, with poly(L-glutamic acid), an uncharged helix is converted 


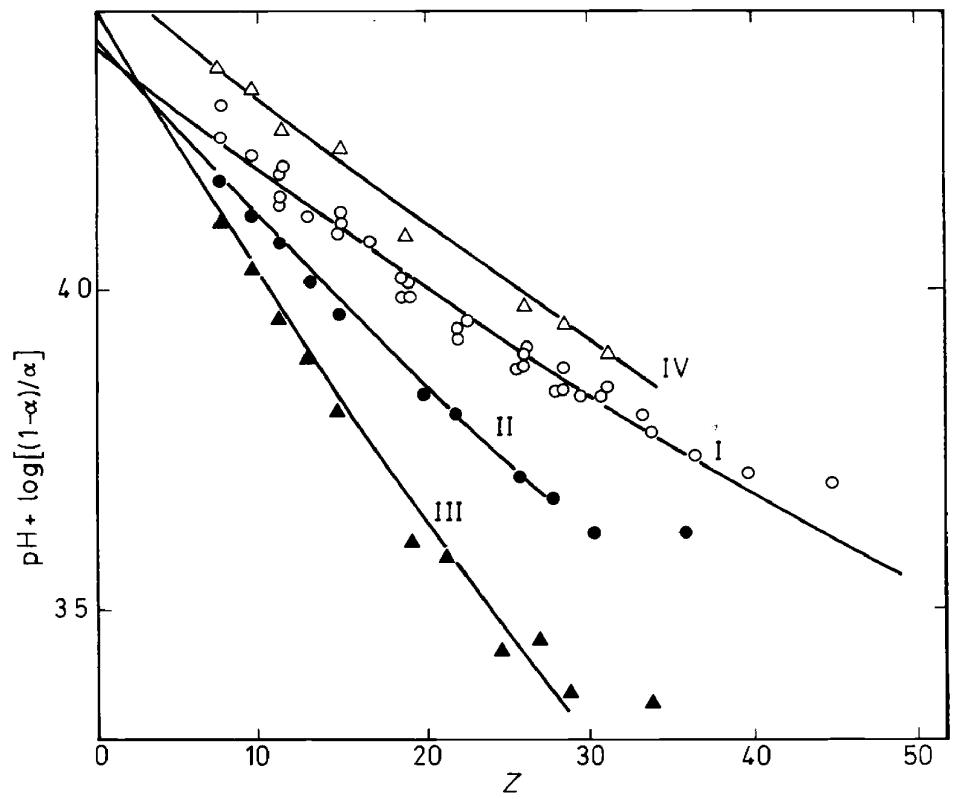

Figure 3a. Potentiometric titration curves for the carboxyl groups of conalbumin. $a=32.0 \AA$.

$\begin{array}{clll}\text { Theor. } & \text { Solvent } & \text { Temp. } & a-b, \\ \text { curve } & \text { KCl } & { }^{\circ} \mathrm{C} & \AA \\ \text { I } & 0.100 \mathrm{M} & 25 & 1 \\ \text { II } & 0.030 \mathrm{M} & 25 & 1.8 \\ \text { III } & 0.010 \mathrm{M} & 25 & 1 \\ \text { IV } & 0.100 \mathrm{M} & 5 & 0.5\end{array}$

The data were obtained by A. Wishnia, I. Weber and R. C. Warner (J. Am. Chem. Soc. 83, 2071 (1961)). (Reproduced, by permission. from ref. 12)

to an uncharged coil. The free energy change $\Delta G$ calculated from the given area is related to the standard free energy change $\Delta G_{a}^{\infty}$ by

$$
\Delta G=\Delta G_{a}^{\infty}+R T \ln (4 C)
$$

The values of $\Delta G_{a}^{\infty}$ so calculated from the area and the total concentration are given in Table $4^{32}$. The average value is $10.0 \mathrm{kcal} / \mathrm{r}$.u.

Comparison between theory and experiment for another protein is shown in Figures $3 \mathrm{a}$ and $3 \mathrm{~b}^{12}$.

\section{(2) Rodlike and coiled polyelectrolytes}

At present, a perfect rodlike polyelectrolyte is not available. Poly(L-glutamic acid) may be the best sample for testing the theory. A comparison between the potentiometric titration data of poly(L-glutamic acid) and the values of $\phi_{b}$ calculated for a rod is shown in Figure $4^{12}$. Agreement is fairly good, but, strictly speaking, the experiments should be repeated more carefully. The experimental data should be extrapolated to infinite dilution for comparison with theory and, moreover, $\mathrm{p} K_{0}$ should be treated as a function of ionic strength. 


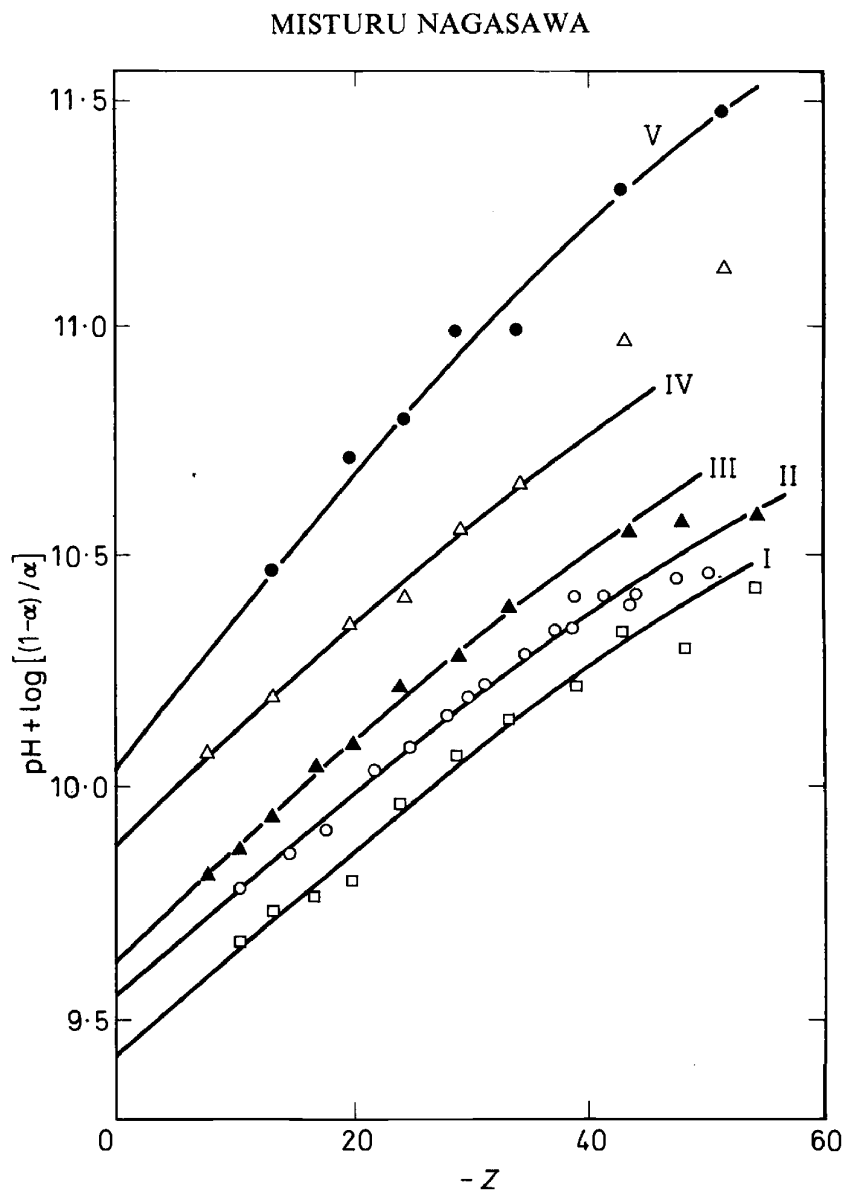

Figure 3b. Potentiometric titration curves for the non-carboxyl groups of conalbumin in 0.100 M KCl. $a=32.0 \AA$

$\begin{array}{ccll}\text { Theor. } & \text { Temp. } & \text { Group } & a-b, \\ \text { curve } & { }^{\circ} \mathrm{C} & \text { titrated } & \AA \\ \text { I } & 25 & \text { Phenolic } & 0 \\ \text { II } & 22 & \text { Phenolic } & 0 \\ \text { III } & 25 & \text {-Amino } & 0.4 \\ \text { IV } & 5 & \text { Phenolic } & 0.6 \\ \text { V } & 5 & \text {-Amino } & 2.4\end{array}$

The data were obtained by the same authors as in Figure 3a. (Reproduced, by permission, from ref. 12).

Table $4 . \Delta G_{0}^{\infty}$ of $\beta$-lactoglobulin by the area method from data for $0.01 \mathrm{M}$ $\mathrm{NaCl}$

\begin{tabular}{ccc}
\hline $\begin{array}{c}\text { Protein conc. } \\
(\%)\end{array}$ & $\Delta G(\mathrm{kcal} /$ r.u. $)$ & $\Delta G_{a}^{\infty}(\mathrm{kcal} /$ r.u. $)$ \\
\hline 0.194 & & \\
\hline 0.469 & 4.7 & 9.7 \\
& 5.7 & 10.2 \\
\hline
\end{tabular}


SYNTHETIC AND NATURAL POLYELECTROLYTES

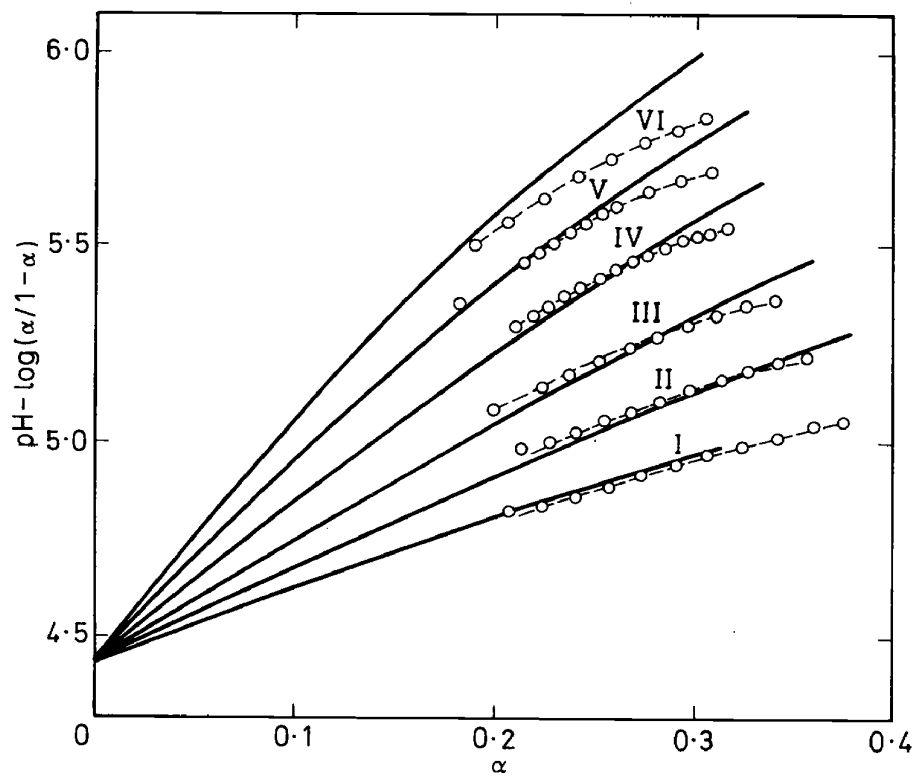

Figure 4. Potentiometric titration curves of helical poly(L-glutamic acid). $a=12.5 \AA, b=0$. PGA conc.: 0.0188 N. Concentrations of $\mathrm{NaCl}$ (moles/1.): I 0.189, II 0.0947, III 0.0475, IV 0.0190, V 0.00954 , VI 0.00478 . (Reproduced, by permission, from ref. 13.)

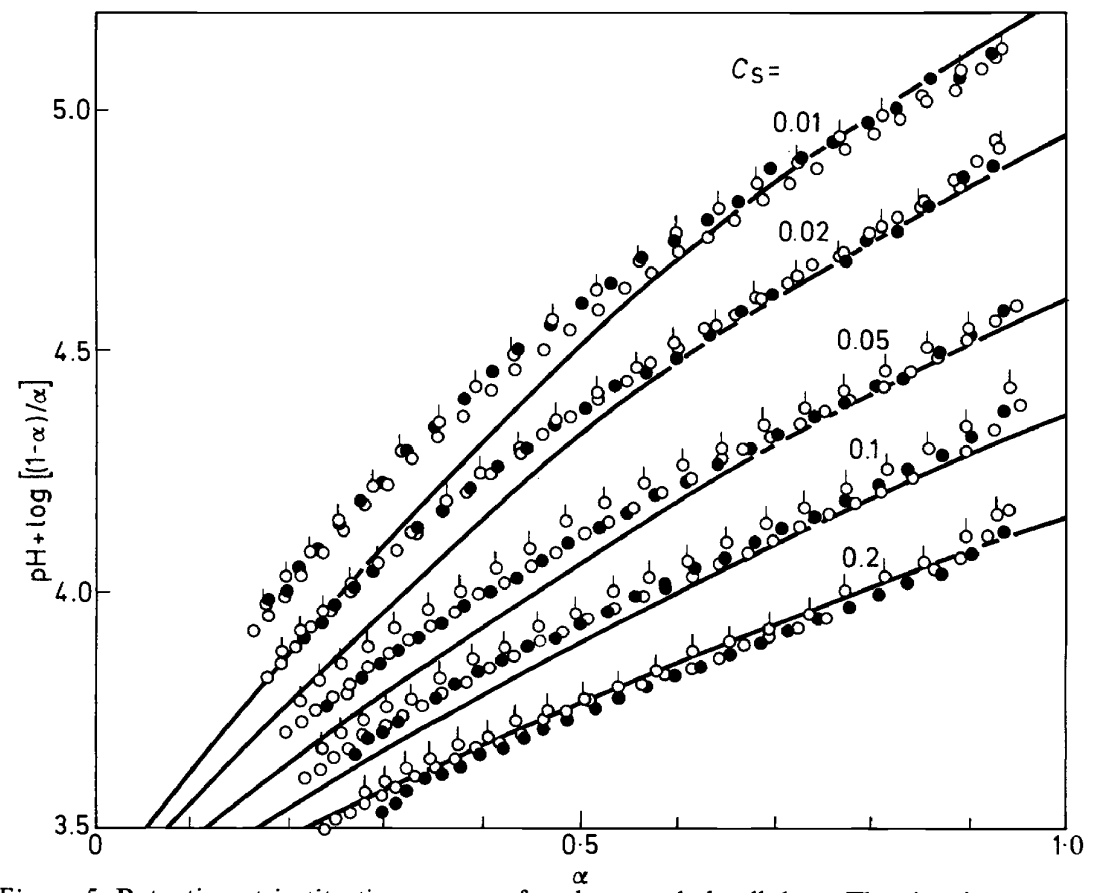

Figure 5. Potentiometric titration curves of carboxymethyl cellulose. The titration was performed at $25^{\circ} \mathrm{C}$ using $\mathrm{NaOH}^{35}$. Sample concentrations: filled circles 0.0084 , open circles 0.0057 , circles with pip $0.0044 \mathrm{~N}$. The concentrations of $\mathrm{NaCl}$ are denoted by $C_{\mathrm{s}}$ in the figure. The thick lines are calculated 


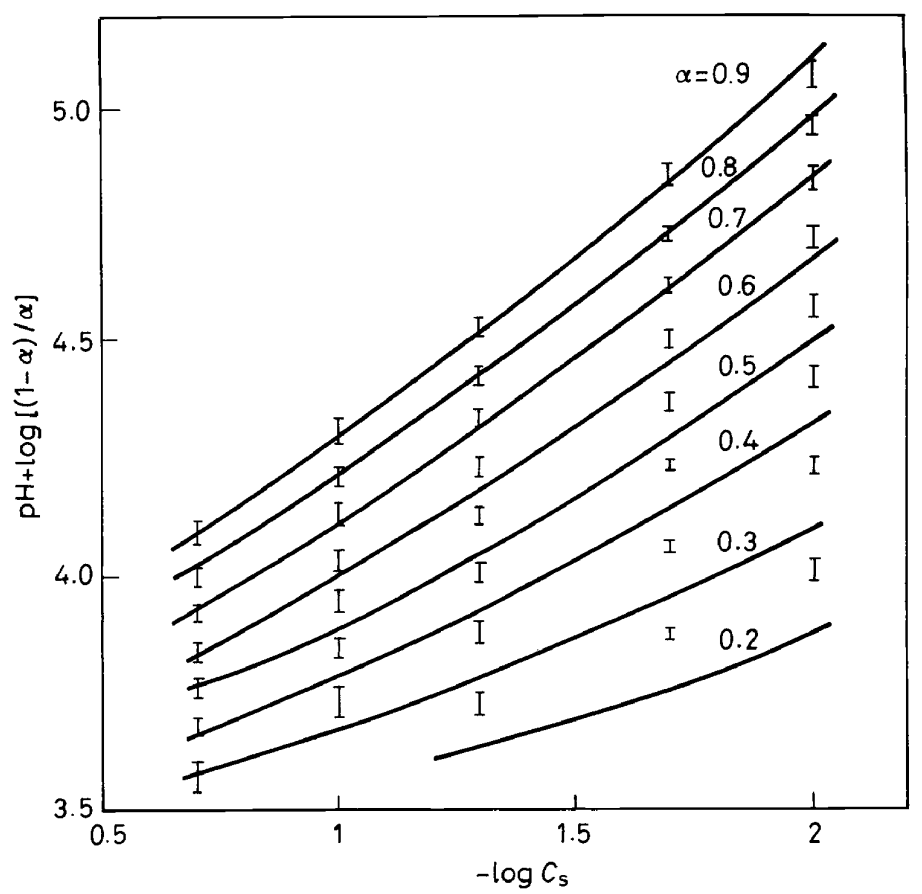

Figure 6. Relationship between $\mathrm{pH}+\log [(1-\alpha) / \alpha]$ and the ionic strength of solvent at various degrees of ionization of carboxymethyl cellulose ${ }^{35}$. The thick lines are calculated. The data are the same as those for Figure 5

It is more difficult to discuss the potentiometric titration curves of linear (flexible) polyelectrolytes because of the flexibility of their backbones. From many studies on polyelectrolyte solutions, however, we can speculate that the potentiometric titration curves of linear polyelectrolytes may be explained by assuming the polyion to be rodlike. In Figure 5, the potentiometric titration curves of carboxymethyl cellulose are compared with the curves calculated for a $\operatorname{rod}^{35}$. The ionic strength dependence of $\mathrm{p} K_{0}$ is taken into account in the calculated values. Agreement between theory and experiments at high degrees of ionization, where the polyion is believed to be fairly extended, is satisfactory but clear disagreement is found at lower degrees of ionization. The conclusion can be confirmed more clearly by plotting the data of $\mathrm{pH}+\log [(1-\alpha) / \alpha]$ against $\log C_{\mathrm{s}}$ at constant degrees of neutralization as shown in Figure 6. The same conclusion was previously reported for poly(acrylic acid) ${ }^{7}$. The plots of $\mathrm{pH}+\log [(1-\alpha) / \alpha]$ versus $1 / \sqrt{ } C_{\mathrm{s}}$ at constant degrees of ionization for poly(acrylic acid) are shown in Figure 7. The disagreement found at low degrees of ionization in Figures 6 and 7 may be attributed to flexibility of the polyion. As the charge density decreases, the expansion factor of the polyion coil decreases so that the rodlike model may become inapplicable. Thus, it may be concluded that the error in estimating the electrostatic free energy change for the melting of DNA would not 


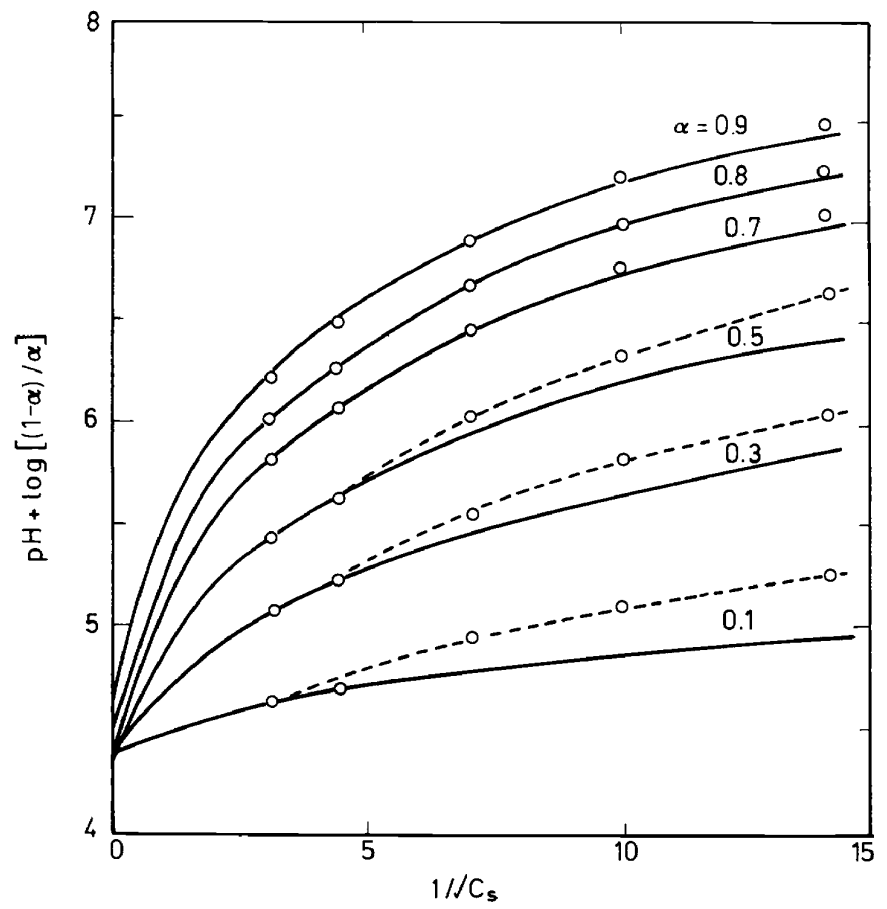

Figure 7. Relationship between $\mathrm{pH}+\log [(1-\alpha) / \alpha]$ and the ionic strength of solvent at various degrees of ionization of poly(acrylic acid). The thick solid lines are calculated. $a=5.5 \AA$. Temperature $15 \pm 2^{\circ} \mathrm{C}$. The degree of ionization is denoted by $\alpha$ in the figure. The data were extrapolated to infinite dilution. (Reproduced, by permission, from ref. 7.)

be so serious even if we assume the denatured polynucleotide to be rodlike.

\section{THE EFFECTS OF LOCAL CONFORMATION ON POTENTIOMETRIC TITRATION OF STEREOREGULAR POLYELECTROLYTES}

From the above conclusion, that the ionization behaviour of linear polyelectrolytes is at least qualitatively explained by using the models of rodlike molecules, one is led to expect that the potentiometric titration curve would depend on the local conformation of the polyion skeleton rather than on the overall shape of the polyion coil. This speculation can be supported by potentiometric titration data of stereoregular polyelectrolytes. That is, because of the strong electrostatic repulsion between fixed charges, the isotactic polyelectrolytes may have a locally helical conformation, whereas atactic and syndiotactic polyelectrolytes may have a planar zig-zag conformation. This difference of local conformation may appear in their potentiometric titration curves. In Figure $8^{7}$, the potentiometric titration curves of isotactic, conventional and syndiotactic poly(methacrylic acids) are compared. It is clear that the isotactic poly(methacrylic acid) has a higher charge density than the syndiotactic one. The same can be observed for poly(acrylic acid), as can be seen in Figure $9^{37}$. 


\section{MISTURU NAGASAWA}

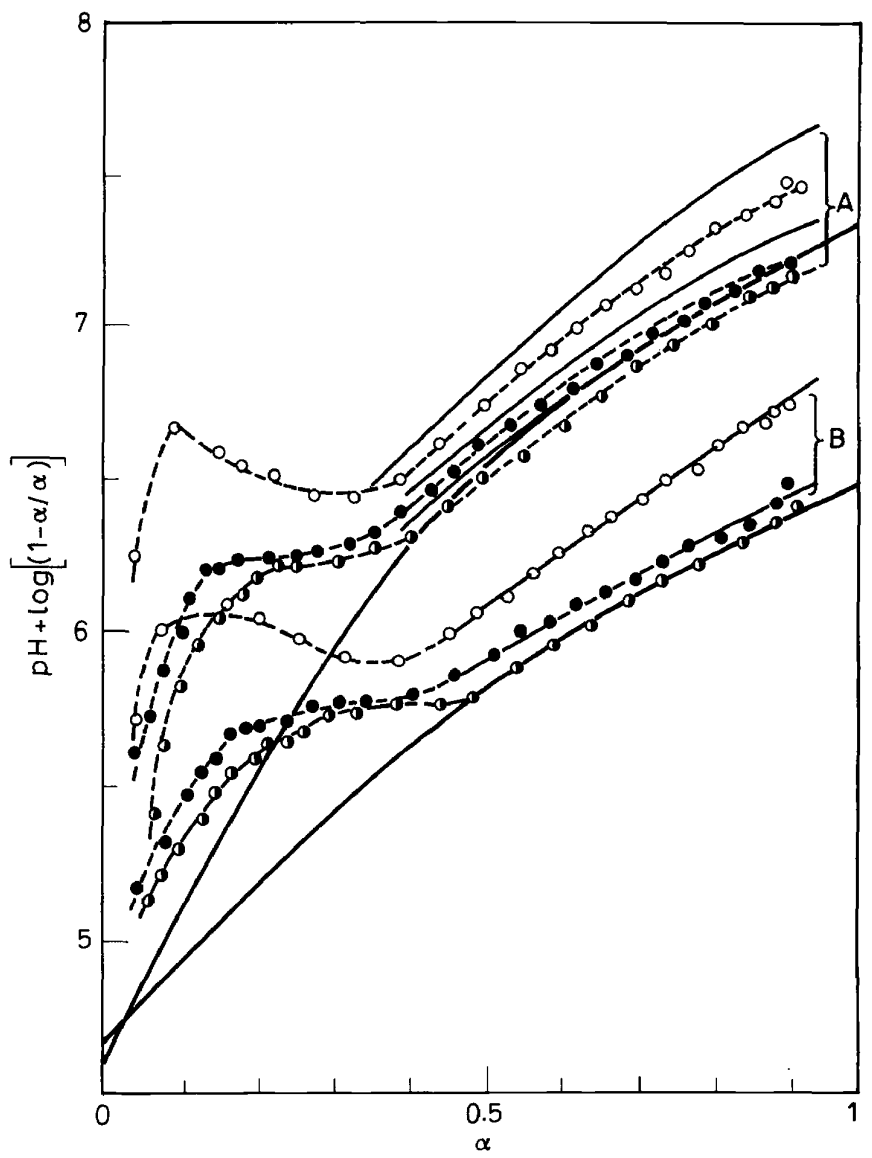

Figure 8. Potentiometric titration curves of stereoregular poly(methacrylic acids). Temperature $23 \pm 2^{\circ} \mathrm{C}$. $\mathrm{NaCl}$ concentrations: A $0.0100, \mathrm{~B} 0.100 \mathrm{~N}$. The open, filled and half-filled circles show experimental data at the lowest polymer concentrations of isotactic, atactic and syndiotactic forms, respectively. The thin solid lines are the values extrapolated to infinite dilution. The thick solid lines are calculated. $a=5.5 \AA$. (Reproduced, by permission, from ref. 7.) 


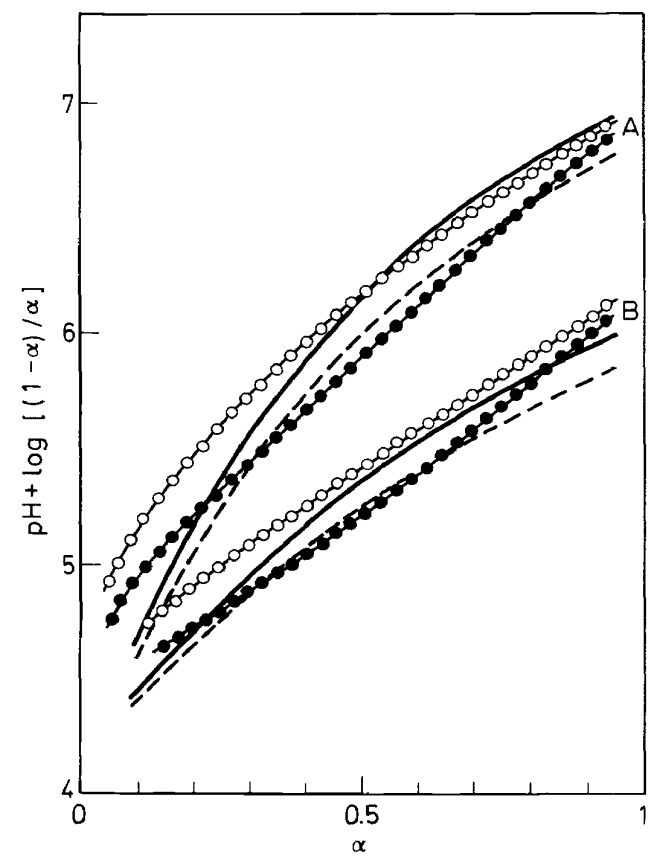

Figure 9. Potentiometric titration curves of isotactic and syndiotactic poly(acrylic acids). Temperature $25 \pm 1^{\circ} \mathrm{C}$. $\mathrm{NaCl}$ concentrations: A $0.0100, \mathrm{~B} 0.100 \mathrm{~N}$. The open and filled circles show experimental data of the isotactic and syndiotactic forms, respectively. The thick solid and broken lines are the calculated ones for isotactic and syndiotactic forms. $a=5.5 \AA$. Degree of helix of the isotactic form is assumed to be 0.72 . (Reproduced, by permission, from ref. 37.)

From proton magnetic resonance spectra of isotactic poly(acrylic acid), we can calculate the coupling constant between the protons in the methylene and methine parts, i.e. vicinal coupling constants, in addition to chemical shifts and the geminal coupling constant. From this vicinal coupling constant, we can calculate the relative positions of protons, that is, the probabilities of trans, gauche and gauche prime forms, from which we can obtain the degree of the helix with an assumption that the probabilities of th, gg and $\mathrm{g}^{\prime} \mathrm{g}$ ' are negligible because of the steric hindrance between sidegroups. The probabilities of $t, g$ and $g^{\prime}$ forms and also the degree of the helix in isotactic poly(acrylic acid) and its sodium forms calculated from those probabilities have been published ${ }^{38}$. The calculated values of $\phi_{b}$ for isotactic poly(acrylic acid) in Figure 9 were obtained by using this degree of helix. On the other hand, the curves of $\phi_{b}$ for syndiotactic poly(acrylic acid) were calculated by assuming the planar zig-zag conformation. The difference between the two calculated curves is comparable to the difference between the two experimental curves, though quantitative agreement between calculated and observed curves is not good because of the flexibility of their backbones.

Muroga, Noda and Nagasawa ${ }^{38}$ reported a constant vicinal coupling constant for isotactic poly(acrylic acid) independent of the degree of neutra- 


\section{MISTURU NAGASAWA}

lization, but Yoshino ${ }^{39}$ showed by using deuterated isotactic poly(acrylic acid) that the vicinal coupling constant and, hence, the degree of helix increases slightly with degree of neutralization. This is in good contrast with the fact that the helical structure of poly(L-glutamic acid) is collapsed by electrostatic repulsion if the molecule is neutralized. Due to the presence of a methyl group, the helical structure of isotactic poly(methacrylic acid) may be more stable than that of isotactic poly(acrylic acid).

\section{REFERENCES}

1 J. Th. G. Overbeek, Bull. Soc. Chim. Belg. 57, 752 (1948).

2 A. Katchalsky and J. Gillis, Rec. Trav. Chim. Pays-Bas. 68, 879 (1949).

3 A. Arnold and J. Th. G. Overbeek, Rec. Trav. Chim. Pays-Bas. 69, 192 (1950).

${ }^{4}$ F. E. Harris and S. A. Rice, J. Phys. Chem. 58, 725 and 733 (1954).

5 G. S. Hartley and J. W. Roe, Trans. Faraday Soc. 36, 101 (1940).

6 A. Katchalsky, N. Shavit and H. Eisenberg, J. Polym. Sci. 13, 69 (1954).

7 M. Nagasawa, T. Murase and K. Kondo, J. Phys. Chem. 69, 4005 (1965).

8 K. Linderstrom-Lang, C.R. Trav. Lab. Carlsberg, Ser. chim., 15, No. 7 (1924).

9 J. Edsall and J. Wyman, Biophysical Chemistry, Academic Press : New York (1958).

10 T. L. Hill, Arch. Biochem. Biophys. 57, 229 (1955).

11 L. Kotin and M. Nagasawa, J. Chem. Phys. 36, 873 (1962).

12 M. Nagasawa and A. Holtzer, J. Am. Chem. Soc. 86, 531 (1964).

13 M. Nagasawa and A. Holtzer, J. Am. Chem. Soc. 86, 538,(1964).

14 B. H. Zimm and S. A. Rice, J. Molec. Phys. 3, 391 (1960).

14 a J. C. Leyte and M. Mandel, J. Polym. Sci. (A-2), 1879 (1964).

15 D. Olander and A. Holtzer, J. Am. Chem. Soc. 90, 4549 (1968).

16 J. Hermans Jr, J. Phys. Chem. 70, 510 (1966).

17 A. Ciferri, D. Puett, L. Rajagh and J. Hermans Jr, Biopolymers, 6, 1019 (1968).

18 R. E. Nylund and W. G. Miller, J. Am. Chem. Soc. 87, 3537 (1965).

19 K. Nitta and S. Sugai, Nippon Kagaku Zasshi, 88, 412 (1967).

${ }^{20} \mathrm{M}$. Nagasawa and $\mathrm{Y}$. Muroga, to be published. There may be a considerable change in discussion.

21 See ref. 7.

22 P. L. Privalov, O. B. Ptitsyn and T. M. Birnstein, Biopolymers, 8, 559 (1969).

23 B. H. Zimm, J.Chem. Phys. 33, 1349 (1960).

24 S. Lifson and B. H. Zimm, Biopolymers, 1, 15 (1963).

25 S. Lifson and B. H. Zimm, Biopolymers, 1, 15 (1963).

26 M. Ozaki, M. Tanaka and E. Teramoto, J. Phys. Soc., Japan, 18, 551 (1963).

${ }^{27}$ E. Teramoto, Seitai Kobunshi, p 245. K. Imahori and F. Oosawa (Eds). Asakura Shoten: Tokyo (1965).

28 J. Marmur and P. Doty, J. Molec. Biol. 5, 109 (1962).

29 L. Schildkraut and S. Lifson, Biopolymers, 3, 195 (1965).

30 W. F. Dove and N. Davidson, J. Molec. Biol. 5, 467 (1962).

${ }^{31}$ M. Tsuboi, Conformation of Biopolymers, Vol. II, p. 689. G. N. Ramachandran (Ed.), Academic Press: New York (1967).

32 M. Nagasawa and A. Holtzer, J. Am. Chem. Soc., in press.

33 R. Townend, L. Weinberger and S. N. Timasheff, J. Am. Chem. Soc. 82, 3175 (1960).

34 S. N. Timasheff, R. Townhend and their co-workers, J. Am. Chem. Soc. 82, 3157, 3161, 3168 (1960); 83, 464, 470, 1419 (1961).

35 Y. Kawaguchi, K. Suzuki and M. Nagasawa, to be published.

36 M. Mandel, Europ. Polym. J. 6, 807 (1970).

37 Y. Kawaguchi and M. Nagasawa, J. Phys. Chem. 73, 4382 (1969).

38 Y. Muroga, I. Noda and M. Nagasawa, J. Phys. Chem. 73, 667 (1969).

39 T. Yoshino, Polymer Symposium at Matsuyama, Japan, 1908. 